\title{
Hydraena (Phothydraena) hernandoi n. sp., nueva especie de España (Coleoptera, Hydraenidae)
}

J. Fresneda 1

A. Lagar ${ }^{2}$

Mots clés : Hydraena hernandoi n. sp., Coleoptera, Hydraenidae, Espagne.

Les auteurs donnent la description d'une nouvelle espèce de Coleoptera Hydraenidae: Hydraena (Phothydraena) hernandoi n. sp. La série type provient de la province de Jaén, Sierras de Cazorla y Segura.

Hydraena (Phothydraena) hernandoi n. sp. a new Species from Spain (Coleoptera, Hydraenidae).

Keywords : Hydraena hernandoi n. sp., Coleoptera, Hydraenidae, Spain.

The authors give the description of a new species of Coleoptera Hydraenidae : Hydraena (Phothydraena) hernandoi n. sp. The typical serie has been collected in the province of Jaén. Sierras de Cazorla y Segura.

Entre el material recolectado por los autores durante el año 1987 en el sur de la Península Ibérica, se ha reconocido como nueva para la ciencia una especie de Coleoptera Hydraenidae perteneciente al subgénero Phothydraena: H. (Phothydraena) hernandoi $n$. sp. En este estudio se incluye más adelante su descripción. El subgénero Phothydraena ha sido objeto de dos estudios del malogrado Dr. C. Berthélemy, con referencias específicas a la fauna ibérica (Berthélemy 1965, 1986). En este último trabajo (Berthélemy 1986) en el que incluye la revisión del subgénero, el autor, al parecer, ya observó algunas diferencias entre los ejemplares determinados como $H$. (P.) testacea Curtis provenientes del sur de España y Marruecos, y los provenientes del norte de la península y Francia. Es probable que la especie que se describe en esta nota sea la misma que ya observó Berthélemy 1986 , refíriéndola este autor a $H$. (P.) testacea Curtis, aunque apunta la necesidad de estudiar más material para poder elaborar el cuadro subespecífico de ésta especie. Sin embargo ante las diferencias observadas y su constancia en numerosos ejemplares - entre $H$. testacea Curtis y $H$. hernandoi $\mathrm{n}$. sp. - se ha juzgado más acertado asignarle identidad específica al nuevo taxon de Andalucía.

1. Escola de Natura de la Vall de Boi, 25527 Barruera, Lleida, España.

2. C/Sanjuanistas 20, 08006 Barcelona, España.
Hydraena (Phothydraena) hernandoi n. sp.

Material estudiado

Holotipo : 1 macho de Jaén, Cazorla, Arroyo de San Pedro de la Garganta, 10.7.1987, Fresneda leg. (en col. Fresneda).

Paratipos : 27 machos y 19 hembras, misma localidad, fecha y recolector que el holotipo $(3 \circ \sigma \mathrm{y}$ $3 \& Q$ en col. Lagar ; 1 o y 1 Q en col. Ferro, Lancenigo ; 1 or y 1 \& en col. del Museu de Zoologia de Barcelona ; 22 or or y 14 $९$ en col. Fresneda).

Material adicional : 1 Q de Málaga, Pujerra, Barranco Hondo, 13.7.1987, Hernando leg.

\section{Descripción}

Biometria del holotipo : longitud máxima - entre el margen anterior del labro y el ápice elitral -1.80 mm. ; anchura máxima - situada a la altura del tercio posterior elitral $-0.70 \mathrm{~mm}$.

Oblongo, de color marrón oscuro (pronoto y élitros) y negro (pronoto y cabeza), con la máxima anchura en el tercio posterior de los élitros.

Cabeza negra. Labro grande con una profunda escotadura en forma de $\mathrm{V}$, puntuado y pubescente, tegumento brillante entre los puntos. Clipeo chagrinado. Frente con la puntuación gruesa y uniformemente repartida, algo más apretada en las proxi- 
midades de los ojos. Ojos de configuración normal, no sobresalen de los ángulos anteriores del pronoto. Palpos maxilares testáceos, con el primer artejo fuertemente arqueado y el último asimétrico (con el margen exterior subrectilineo y el interior regularmente convexo).

Pronoto alargado, marrón oscuro con una ancha franja transversal negra. Margen anterior sinuoso, posterior recto, laterales subparalelos en la primera mitad, con suave curva (no ángulo marcado) en la mitad, y algo cóncavos y convergentes entre si en el tercio posterior. Margen posterior más estrecho que la base de los élitros. Existen cuatro foveolas, dos a cada lado del disco, una anterior y otra posterior. Puntuación gruesa, densa y regularmente repartida. El escaso tegumento visible entre los puntos brillante.

Élitros marrón oscuro, con la máxima anchura en el tercio posterior. Rebordeados, y éste reborde denticulado. Existen siete series de puntos bien definidas entre la sutura y el callo humeral. Los puntos grandes, perfectamente alineados y en muchas ocasiones en contacto entre sí. Las interestrias muy estrechas, de recorrido sinuoso entre dos filas de puntos. Los puntos de la serie marginal más grandes que los demás y incrementando su tamaño cuanto más cerca del ápice, y en estos últimos el interior ocupado por una membrana traslúcida.

Parte inferior negra. Metaesternón y metacoxas micropuntuadas densamente, de aspecto rugoso y mate. Placas lisas metasternales en número de cuatro : dos largas y estrechas confluyentes en la parte anterior (en forma de $\mathrm{V}$ invertida), y dos pequeñas (muy estrechas y cortas) al exterior de éstas.

Patas de color testáceo oscuro. Tibias rectas, por lo demás absolutamente normales.

Edeago - en vista dorsal (según fig. 1) - alargado, con el lóbulo medio ancho, truncado y con una pequeña prolongación lateral truncada ; una laminilla espicular de recorrido curvado en el ápice (fig. 1 AD) y dos cortas quetas romas en forma de maza insertadas en el lateral (fig. 1 Q). Parámeros gruesos, insertados en la parte ventral del lóbulo basal ; alcanzan en su recorrido el ápice del lóbulo medio ; con tres grupos de sedas : uno distal con 5-7 sedas (fig. $1 \mathrm{SD}$ ), uno medio con dos sedas (fig. $1 \mathrm{SM}$ ) y uno proximal con 4-5 sedas ( $f i g .1 \mathrm{SP}$ ). El edeago en vista lateral según figura 2.

Variaciones

Los ejemplares estudiados son absolutamente constantes para la descripción del holotipo. El edeago fue extraido y montado de 28 machos, revelandose como una extructura constante.

\section{Dimorfismo sexual}

El reborde elitral es algo más acusado en las hembras. Noveno esternito abdominal de la hembra según figura 5.

\section{Derivatio nominis}

Especie dedicada el colega C. Hernando de Barcelona, con quien se localizaron los ejemplares de la serie típica.

\section{Discusión}

La máxima afinidad de ésta nueva especie se encuentra en $H$. (P.) testacea Curtis, con quién comparte morfologia externa (aunque son diferenciables sin necesidad de recurrir al estudio del edeago) y similar modelo, en líneas generales, de edeago. Ya Berthélemy 1986 en su revisión del subgénero Phothydraena, comenta haber estudiado ejemplares del sur de España y Norte de África, y dice haber observado diferencias respecto de la $H$. (P.) testacea Curtis, común en el norte de España y Francia : "The specimens from southern Spain and N. W. África are more heavily pigmented. Their parameres and the proximal piece of their aedeagus are more narrow than in specimens from northern Spain and France. In some localities (Sierra de Segura, Spain ; Ceuta, Morocco), the curved setae of the proximal piece are shorter. Much more material would be needed to decide whether these intraspecific variations are clinal or whet her subspecies may be distinguished within $H$. testacea " (Berthélemy 1986).

Sin embargo, la valoración de las diferencias observadas respecto de $H$. testacea Curtis y su constancia en todos los ejemplares estudiados permite conceder - a juicio de los autores - categoría especifica a $H$. hernandoi $\mathrm{n} . \mathrm{sp}$.

Estas dos especies $H$. testacea Curtis y $H$, hernandoi $\mathrm{n}$. sp. se separan con facilidad por los siguientes caracteres : $H$. hernandoi n. sp. es más oscura que $H$. testacea Curtis ; los puntos foveolados de la serie marginal son considerablemente más grandes en $H$. testacea Curtis, y en ocasiones el último está horadado, más pequeños (comparables a los de $H$. atrata Desbrocher des Loges), y nunca horadados 

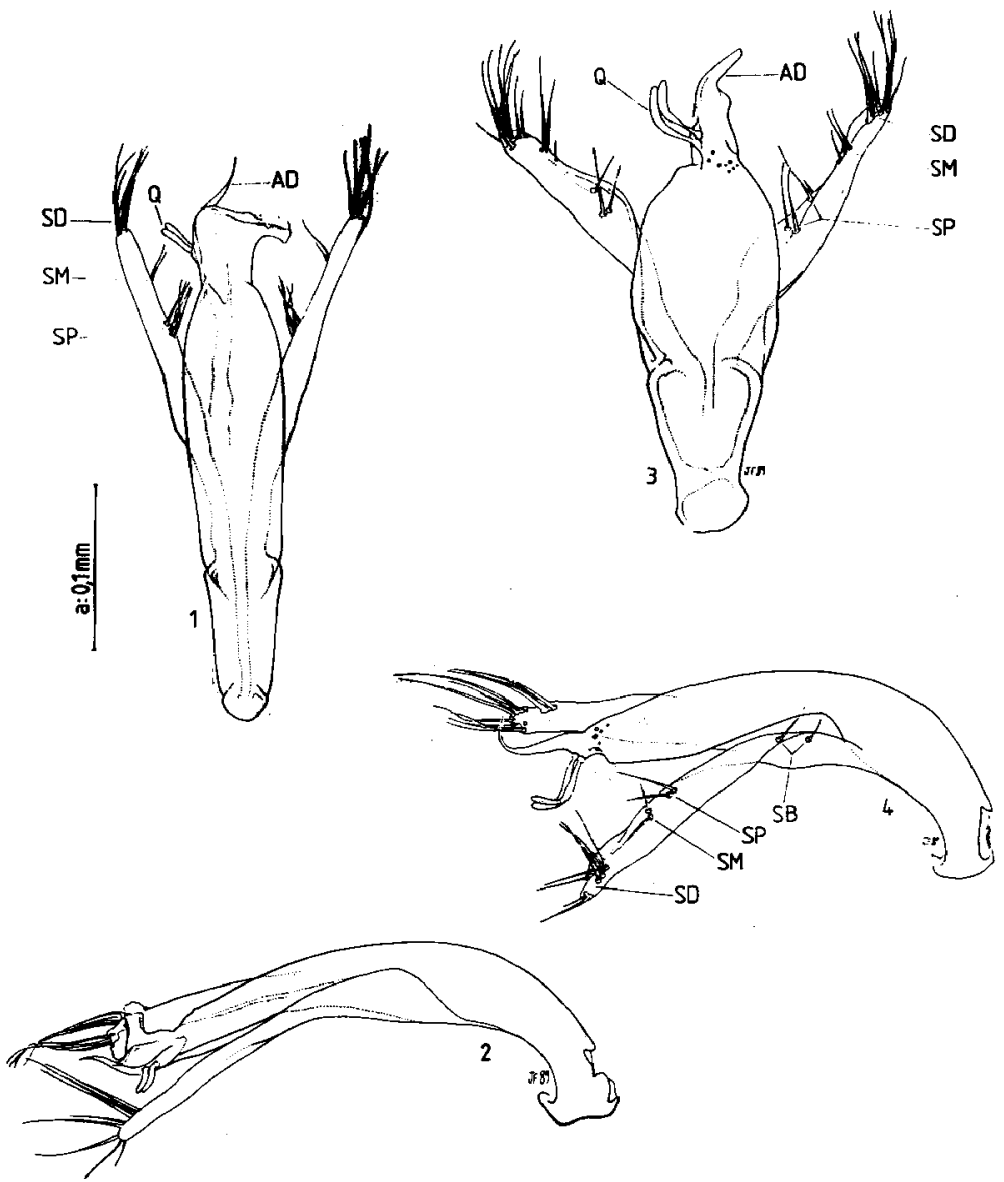

Fig. 1-4 : Edeago de : 1. H. hernandoi n. sp., vista dorsal ; 2. la misma especie, vista lateral ; 3. H. testacea Curtis de Sant Cugat (Barcelona), vista dorsal ; 4. la misma especie, vista lateral. Lóbulo medio : AD, apéndice distal ; $Q$, quetas laterales del lóbulo medio ; parámeros : SD, grupo de sedas distales ; SM, grupo de sedas medio ; SP, grupo de sedas proximal ; SB, grupo de sedas basal. La escala « a " corresponde a las figuras 1-4. 
en $\boldsymbol{H}$. hernandoi $\mathrm{n}$. sp. El protórax en $H$. testacea Curtis es algo más rectangular ; en $H$. hernandoi $\mathrm{n}$. sp., parece estrecharse un poco, gradualmente, desde la base hasta el borde anterior.

En cuanto al edeago, para separar $H$. testacea Curtis de $\boldsymbol{H}$. hernandoi n. sp., se remite a la descripción del edeago del holotipo de ésta última especie para poder comparar con la siguiente de $H$. testacea Curtis (figs. 3-4 ; d'Orchymont 1930, fig. 28 ; Berthélemy 1965 p. 6, figs. 1-2) : lóbulo basal ancho y corto (muy robusto) ; el lóbulo medio más largo que ancho, prolongado por un apéndice curvado romo o truncado (fig. $3 \mathrm{AD}$ ) ; dos largas quetas curvadas y romas insertadas en el lateral (fig. $3 \mathrm{Q}$ ) ; un pequeño grupo de sensilios en la base del lóbulo medio. Parámeros robustos, insertados ventralmente con tres-cuatro grupos de sedas : uno distal muy numeroso compuesto por $9-10$ sedas (fig. 3 SD) ; uno medio con $2-3$ sedas (fig. 3 SM) ; y otro proximal, quizá dividido en dos formado por I + 2 o $2+2$ sedas (fig. $3 \mathrm{SP}$ ).

Esto grupos son equivalentes a los que se encuentran en $H$. hernandoi $\mathrm{n}$. sp. ; pero en $H$. testacea Curtis existe otro grupo formado por dos sedas, cerca de la base del parámero (fig. $4 \mathrm{SB}$ ). El edeago en visión lateral según la figura 4 . También se observan diferencias en la conformación del último esternito abdominal de la hembra: la figura 5 corresponde al de $H$. hernandoi n. sp., y la 6 al de $H$. testacea Curtis.

De $H$. testacea Curtis se ha estudiado el siguiente material : 1 ej. Barcelona, Borredà, Riera de Merlés, 28.7.1982, Fresneda leg. ; 8 ej. Barcelona, Sant Cugat, Can Borrell, 6.7.1986, Fresneda leg. ; 8 ej. Barcelona, Vallvidrera, Riera del Pantà, 11.77.1988, Fresneda leg. ; 1 ej. Cádiz, Los Barrios, Arroyo del Tiradero, 13.7.1987, Fresneda leg. ; 3 ej. Cádiz, Grazalema, Arroyo del Caserio el Pinar, 15.7.1987, Fresneda leg. ; 1 ej. Cádiz S ${ }^{\mathrm{a}}$ Grazalema, Benamahoma, Fuente del Descansadero, Agosto 1985, Fresneda leg. ; 1 ej. Girona, Castelló d'Ampuries, 8.8.1984, Fresneda leg. ; 1 ej. Jaén, Cazorla, Arroyo de Valdecuevas, 10.7.1987, Fresneda leg. ; 3 ej. Málaga, Cortes de la Frontera, Garganta de Rosada Llana, 14.7.1987, Fresneda leg. ; 3 ej. Tarragona, Arnes, Riu Algars, 12.8.1986, Fresneda leg.

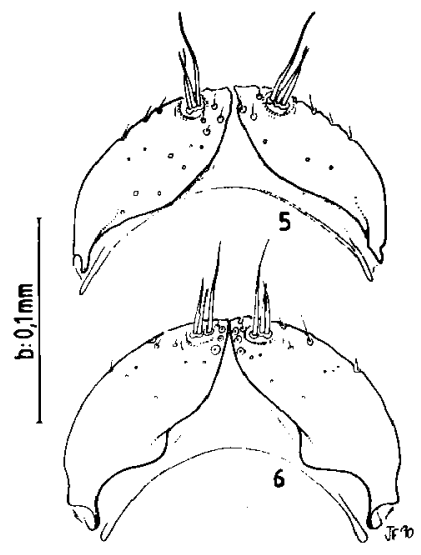

Fig. 5-6 : Ültimo esternito abdominal de la hembra de : 5. H. hernandoi n. sp. ; 6. H. testacea Curtis de Vallvidrera (BARCELONA). La escala $* \mathbf{b}$ » corresponde a las figuras $5-6$.

\section{Distribución y ecología}

Por el momento $-H$. hernandoi $\mathrm{n}$. sp. - confinada al sureste peninsular, Sierra de Cazorla y Sierra Bermeja, aunque probablemente, a juzgar por los datos de Berthélemy 1986, se encuentre bastante extendida por el sur de la Península Ibérica e incluso por el Magreb (Marruecos).

Por las localidades en que se ha recolectado $H$. testacea Curtis, presumiblemente extendida por toda o gran parte de la geografía ibérica. Incluso se han estudiada ejemplares de una localidad vecina del enclave de la serie típica de $H$. hernandoi $\mathrm{n}$. sp. Es interesante comentar, que los ejemplares de $H$. testacea Curtis estudiados de localidades del sur peninsular, son algo más oscuros que los recolectados en el norte.

En cuanto a las características del arroyo de San Pedro de la Garganta (localidad tipica), cabe apuntar que es un curso de agua permanente, aunque la parte alta llega a secarse en periodo estival. En este caso sólo quedan charcas aisladas en el cauce, o con 
infima corriente de agua que las une. Es en este tipo de biotopo donde se recolectó $H$. hernandoi $\mathrm{n}$. sp. Se recolectaron en el mismo lugar las siguientes especies de Hydraena : $H$. (Hadrenya) servilia d'Orchymont, $H$. (s. str.) subdepressa Rey, $H$. (s. str.) cordata cordata Schaufuss, $H$. (s. str.) quilisi Lagar, Fresneda \& Hernando, e $H$. (s. str.) capta d'Orchymont.

\section{Bibliografia}

Berthélemy (C.). 1965. - Note taxonomique et faunistique sur des Hydroena Françaises et lbériques (Coléoptères). Annis. Limnol. 1 (1) : 3-19.

Berthélemy (C.). 1986. - Remarks on the genus Hydraena and revision of the subgenus Phothydraena (Coleoptera : Hydraenidae). Annls. Limnol. 22 (2) ; 181-193.

Orchymont (A. d'). 1930. - A propos de la Tyrrhénide. Les Hydraena. Mém. Soc. Ent. Belgique $23: 33-49$. 